\title{
Implementación de un modelo educativo y de concientización para promover la cultura de seguridad en los hospitales de la Secretaría de Salud, Hidalgo, México
}

Implementation of an Educational and Awareness Model to Promote Safety Culture Inside the Hospitals of the Ministry of Health, Hidalgo, Mexico

\author{
Marco A. Escamilla Acosta ${ }^{a}$, José María Busto Villarreal ${ }^{b}$, Gloria Samantha
}

Mendoza Jiménez ${ }^{c}$, Reyna Villegas García ${ }^{\text {, Carlos Montaño Salazar }}{ }^{e}$

\begin{abstract}
:
Patient safety is a priority at any level of health care, each stakeholder involved in the process serves as a key element for efficient care, providing quality, safe, and on time procedures that generate key indicators for the development of our state and our country. Multiple actions have been carried out to highlight the importance of this issue, small nuclei actions to even large specialized care units, relate particular efforts that generate large-scale results and that directly impact in statistics at the state, national and global levels that reflect the commitment to a universal right: health. Generating a program that standardizes and promotes the culture of safety in the procedures carried out in a second and third level of care, consolidates a strategy of the Health Ministry in the State of Hidalgo with its 16 hospitals distributed within the State, this strategy, encourages commitment to safe, efficient and quality care. The development of this article, will focus on the educational and quality management strategy implemented, which aims to join efforts and align policies with the vision of zero preventable deaths of the Patient Safety Movement, a movement with the participation of 43 countries.
\end{abstract}

\section{Keywords:}

Patient safety, quality, education, awareness

\begin{abstract}
Resumen:
La seguridad del paciente es prioritaria en cualquier nivel de atención a la salud, cada involucrado en el proceso funge como una pieza clave para una atención eficiente, el ofrecer atenciones y procedimientos seguros y a tiempo corresponden a una prestación de servicio de calidad, generando indicadores clave para el desarrollo de nuestro estado y de nuestro país. Múltiples acciones se han llevado a cabo para resaltar la importancia de este tema, las acciones en los núcleos pequeños hasta en grandes unidades de atención especializada, corresponden a esfuerzos particulares que generan resultados de gran magnitud y que impactan directamente en estadísticas a niveles estatal, nacional y mundial que reflejan el compromiso con un derecho universal: la salud. El generar un programa que estandarice e impulse la cultura de la seguridad en los procedimientos llevados a cabo en un segundo y tercer nivel de atención, consolida una estrategia de la Secretaría de Salud en el Estado de Hidalgo con sus 16 hospitales distribuidos en el interior del Estado, que fomenta el compromiso con una atención segura, eficiente y de calidad. En el desarrollo del presente artículo se dará a centra en conocer la estrategia educativa y de gestión de calidad implementada, cuyo objetivo es sumar esfuerzos y alinear políticas a la visión de cero muertes prevenibles del Patient Safety Movement, movimiento con participación de 43 países.
\end{abstract}

Palabras Clave:

Seguridad del paciente, calidad, educación, concientización

\section{Introducción}

La Organización Mundial de la Salud, durante su Asamblea Mundial de la Salud 2002 adoptó la seguridad del paciente como parte de sus resoluciones y pone en marcha en 2004, junto con 140 países algunos programas para solucionar los problemas que demandan una atención con garantía de seguridad. Tales iniciativas cobran relevancia tras conocer que en países desarrollados hasta uno de cada diez pacientes

\footnotetext{
a Secretaría de Salud del Estado de Hidalgo, Dirección General de los Servicios de Salud, Email: marco.escamilla@ssh.gob.mx

Autor de correspondencia, Secretaria de Salud del Estado de Hidalgo, Dirección de Segundo Nivel de Atención, Email: jose.busto@tuzos.com.mx

' Secretaría de Salud del Estado de Hidalgo, Dirección de Segundo Nivel de Atención, ORCID: https://orcid.org/0000-0002-6809-4168, Email: gsamanthamj@gmail.com

d Secretaría de Salud del Estado de Hidalgo, Dirección de Segundo Nivel de Atención, Email: apv_direccionhospitales@outlook.com

e Secretaría de Salud del Estado de Hidalgo, Subdirección de Acreditación y Certificación, Email: cmontanosalazar@gmail.com
} 
sufre algún daño durante su estancia en hospitales; de cada 100 pacientes hospitalizados, 7 en países desarrollados y 10 en países en vías de desarrollo contraerán infecciones relacionadas con la atención de la salud, sin embargo, la frecuencia de tales infecciones puede reducirse en más de un $50 \%$ con la adopción de medidas sencillas; existen 1.5 millones de dispositivos médicos diferentes, la mayor parte de la población no tiene acceso adecuado, más de la mitad no cuenta con normativa nacional sobre tecnología sanitaria; que cada año se realizan 234 millones de operaciones quirúrgicas, que conllevan un riesgo considerable, de las complicaciones en las cirugías, el $50 \%$ pueden evitarse; hay un mal uso del gasto en salud entre el $20 \%$ y el $40 \%$ se desaprovecha a causa de una atención deficiente; la probabilidad de que un paciente pueda sufrir algún evento adverso en una unidad de atención a la salud es de 1 en 300 (OMS 2, 2018).

Todo el mundo, se enfrenta a un desafío para evitar accidentes $u$ omisiones de seguridad en la atención de la salud, como personal de salud tenemos como estandarte el "Lo primero es no hacer daño", razón por la cual y como lo menciona la Joint Comission: lo primero es reconocer la realidad que enfrentamos, rechazar el statu quo que es aceptable y tomar medidas para corregir los problemas que contribuyen a la atención no segura (OMS 2, 2007).

Actualmente, las soluciones para mejorar la seguridad del paciente han trascendido y tienen un enfoque más constructivo, en donde se involucra con mayor fuerza el trabajo en equipo, la comunicación efectiva tanto entre el personal de salud como con los pacientes y la unificación de procesos de atención, ha llegado el momento de crear y difundir más que la problemática, las soluciones para ir rumbo a la prevención de atenciones deficientes (OMS 2, 2007).

Múltiples alianzas han surgido tras la publicación de las problemáticas expuestas previamente, cuyo objetivo principal es coordinar, difundir y acelerar las mejoras estratégicas para fomentar la seguridad del paciente en todo el mundo. En 1951 se funda la Joint Comission cuya visión es que las personas experimenten atención médica más segura, de mayor calidad y con el mejor valor (The Joint Comission, 2018); en 2005 la OMS lanzó la Alianza Mundial para la Seguridad del Paciente e identificó 6 campos de acción (OMS 2, 2007); en 2012 en Estados Unidos de América, nace el Patient Safety Movement, un movimiento para reunir a todos los involucrados en la seguridad del paciente (Patient Safety Movement, 2018). En México, como país integrante de esta Alianza, en el año 2017 el Consejo de Salubridad General en México a través del Diario
Oficial de la Federación publica el acuerdo para que todos los integrantes del Sistema Nacional de Salud consideren como obligatorias las "Acciones Esenciales para la Seguridad del Paciente" (DOF, 2017).

Específicamente, las acciones esenciales del paciente en México son: identificación del paciente, comunicación efectiva, seguridad en el proceso de medicación, seguridad en los procedimientos, reducción del riesgo de infecciones asociadas a la atención a la salud, reducción de riesgo por caídas, registro y análisis de eventos centinela, adversos y cuasi fallas y cultura de seguridad del paciente (SSA, 2018).

\section{Desarrollo}

Dado que la seguridad del paciente es un reto y compromiso mundial y que México forma parte de la Alianza, la Secretaria de Salud del Estado de Hidalgo lleva a cabo acciones constructivas que impulsan la seguridad del paciente, comprometida con la mejora de la calidad en cuanto a la atención a la salud, ha forjado objetivos cuyos ejes estratégicos son las acciones esenciales del paciente.

El Patient Safety Movement es un movimiento internacional que incluye a 43 países, su esquema de participación consiste en el alta de compromisos anuales, tiene como objetivo: cero muertes prevenibles y se alinea a las acciones esenciales de seguridad del paciente; como estrategia en la $\mathrm{SSH}$, en enero de 2018, se dan de alta en la plataforma de Patient Safety Movement (www.patientsafetymovement.org) a los 16 Hospitales de la Secretaría de Salud en Hidalgo: Hospital Regional del Valle del Mezquital, de la Huasteca, Hospitales Generales de Pachuca, Tula, Actopan, Huichapan, Apan, Tulancingo, Obstétrico, Villa Ocaranza y Hospitales Integrales Atlapexco, Jacala, Cinta Larga, Tlanchinol, Huehuetla y Otomí Tepehua, con 2 compromisos o Soluciones Accionables por la Seguridad del Paciente (APSS, por sus siglas en inglés) que van alineados a las principales carencias identificadas dentro de nuestra institución: a) Cultura de la Seguridad (APSS 1) y b) Optimización de la Seguridad Obstétrica (APSS 11).

La Secretaría de Salud, ha forjado objetivos cuyos ejes estratégicos son acciones esenciales para mejorar la calidad de la atención y cultura de seguridad del paciente.

\section{La estrategia:}

Dada la necesidad de implementar mejoras en los procesos de atención, fue menester evaluar el 
conocimiento de fortalezas, oportunidades, deficiencias y amenazas de nuestro sistema $y$ establecer herramientas para identificar las carencias y necesidades en todos los ámbitos involucrados (recursos, tiempo, costos e infraestructura), factor clave para el planteamiento de la solución real a implementar en favor de la seguridad del paciente en colaboración con los grupos de trabajo de los sectores involucrados, el objetivo principal: hacer planes de acción documentados e innovadores, generar medidores de avance; a partir de esta iniciativa, fortalecer los programas ya existentes consolidó el plan estratégico a llevar a cabo con la finalidad de homogenizar procesos y aprender de los errores, tomando en consideración las experiencias exitosas de otros estados; lo anterior encaminado a la estrategia nacional.

\section{La planeación:}

Durante el proceso de alta de compromisos en la plataforma se definieron los retos a comprometer alineados a la estrategia del Patient Safety Movement y sus lineamientos publicados (www.patientsafetymovement.org), fue prioritaria la evaluación de condiciones de los hospitales y su factibilidad de implementación a través de la unificación de conocimientos para lograr el planteamiento de estrategias en el tiempo y alcance. En lo particular, cada Hospital dentro del marco general, buscó diseñar y adaptar el modelo, para constituir acciones de acuerdo a las necesidades, deficiencias de sus servicios e incidencias de error de atención más comunes. Estos ejes principales bajo los cuales se fomenta la seguridad del paciente son:

1. Promoción, concientización y capacitación de forma regional y particular por hospital,

2. Definición de líneas prioritarias en seguridad del paciente,

3. Definición de indicadores clave de desempeño,

4. Reporte de eventos adversos,

5. Tablero de control para seguimiento a los eventos adversos.

Los ejes anteriores se apoyan del Sistema Tecnológico de Registro de eventos adversos, software que es provisto por la Dirección General de Calidad en Salud (DGCES) y funge como recurso tecnológico principal para poder hacer el registro detallado de tales eventos con la intención de estandarizar las estrategias a llevar a cabo para mejorar las condiciones de seguridad de atención.

\section{La estratificación de información.}

La cultura de seguridad del paciente así como la optimización de la seguridad obstétrica, debe darse a conocer a todo el personal que se encuentre directa o indirectamente relacionado con la atención del paciente, para ello personal médico, administrativo y de soporte de operaciones atendió a reuniones regionales de capacitación, el objetivo: compartir con cada involucrado de la atención a la salud, la necesidad de una cultura de la seguridad en todos los servicios de los hospitales, hacer énfasis en la responsabilidad que recae en cada momento y factor involucrado en el proceso de atención y la necesidad de una atención fiable y segura de las personas que demandan un servicio, asimismo se consideraron algunos conceptos legales que demandan o defienden las acciones de personal de atención a la salud así como el análisis de errores a través del reporte de eventos adversos. Tales reuniones se llevaron a cabo durante el mes de marzo, 2018 e incluyeron a las regiones: Tulancingo (Hospital Tulancingo, Huehuetla, Otomí Tepehua y Apan); Región Ixmiquilpan (Hospital Valle del Mezquital, Jacala, Actopan y Huichapan); Región Huejutla (Hospital Huejutla, Tlanchinol y Atlapexco); Región Tula (Hospital Tula, y Cinta Larga) y Región Pachuca (Hospital Pachuca, Obstétrico, Villa Ocaranza y Oficinas centrales). En lo posterior se llevaron a cabo capacitaciones en diversos eventos, tales como el Congreso de Enfermería, Comités de seguridad del paciente, Aniversarios de Hospitales y se aventaja del uso de la tecnología de teleeducación a través de medios digitales.

Tras haber dado a conocer la importancia de la seguridad del paciente durante el primer semestre 2018, se logró capacitar presencial como virtualmente a 4800 personas en temas relacionados con la cultura de la seguridad del paciente, tales como: Lavado de manos, Cultura de seguridad del paciente, Sistema de eventos adversos, Acciones esenciales de seguridad del paciente, Indicadores de calidad en salud, Infecciones asociadas a la atención de la salud, Expediente clínico, Uso de medicamentos, Atención al paciente en estado crítico, RPBI, Carro rojo, Cédula de acreditación con enfoque en seguridad del paciente, Registros clínicos de enfermería, Riesgos de caídas, Reducción de cesárea, Triage obstétrico, GPC de parto, cesárea y puerperio, Paciente obstétrica, Código mater y Protección de vida del binomio.

Como parte del proceso de capacitación al personal de atención a la salud se implementó una plataforma educativa a distancia sobre el dominio Moodle, 
denominada: Movimiento por la seguridad de la paciente, misma que alberga la teleducación de 128 personas, con 2 profesionales de la salud como ponentes, los módulos que contempla son: seguridad del paciente, soluciones para la seguridad del paciente y eventos adversos.

Durante el segundo semestre se llevó a cabo una segunda serie de capacitaciones regionales, cuya finalidad fue el seguimiento continuo y el análisis de los errores identificados a través del análisis de causa raíz. Las capacitaciones en cuanto a la importancia de la seguridad, así como al uso de los recursos tecnológicos para su aprovechamiento, trajo como resultado el reporte de 231 eventos adversos del mes de enero a diciembre de 2018.

\section{Discusión}

Las evaluaciones de cultura de seguridad, son herramientas que pueden ser utilizadas para medir condiciones organizativas que conducen a eventos adversos y daño al paciente; así como para desarrollar y evaluar la seguridad. Intervenciones de mejora en los hospitales proporcionan una métrica de entendimientos compartidos implícitos que pueden modelo, en la fase de capacitación y concientización de los profesionales de atención a la salud del segundo nivel, es el incremento considerable de reporte de eventos adversos, centinelas y/o cuasifallas, de 39 (2016), 88 (2017) a 231 (2018), lo que a su vez permitirá incrementar la seguridad del paciente y reducir la mortalidad en las pacientes obstétricas.

\section{Conclusiones}

La primera fase de la implementación del modelo para la promoción de la cultura de seguridad en el interior de los hospitales en el Estado de Hidalgo, corresponde a una tarea que involucró herramientas para un proceso educativo y de concientización de la cultura de seguridad del paciente, misma que cobra relevancia para la atención de daños a la salud que previenen el incremento en la mortalidad y a su vez acrecientan calidad en la atención a través del aprendizaje de los errores de los involucrados.

La ejecución de capacitaciones regionales y a nivel local, fue pieza fundamental para permear de información valiosa a profesionales de la atención a la salud que tienen contacto directo o indirecto con los pacientes. Fue prioritario que cada unidad hospitalaria contara con un plan estratégico adecuado a las necesidades de los servicios de sus unidades médicas. El análisis y puesta en marcha de programas de prevención y modelos de gestión de calidad permite visualizar una atención de mayor calidad conforme se incrementa la curva de aprendizaje. El conocimiento del impacto de la seguridad del paciente, así como su participación en el proceso de aprendizaje y creación de una cultura de seguridad a nivel general y en el ámbito obstétrico, serán la base para transforma la cultura organizacional de los hospitales, en específico el reporte continuo de incidentes para mejorar la atención médica y prevenir el incremento de mortalidad.

\section{Referencias}

[1] Diario Oficial de la Federación, DOF. (2017). Acuerdo por el que se declara la obligatoriedad de la implementación, para todos los integrantes del Sistema Nacional de Salud, del documento denominado Acciones Esenciales para la Seguridad del Paciente. 5 de noviembre de 2018, de Vlex México.

[2] Organización Mundial de la Salud, OMS. 1 (2007). Preámbulo a las soluciones para la seguridad del paciente. 5 de noviembre de 2018, de Organización Mundial de la Salud.

[3] Organización Mundial de la Salud, OMS. 2 (2018). 10 Datos sobre Seguridad del Paciente. 5 de noviembre de 2018, de Organización Mundial de la Salud.

[4] Organización Mundial de la Salud, OMS. 3 (2018). Seguridad del paciente. 5 de noviembre de 2018, de Organización Mundial de la Salud

[5] Patient Safety Movement. (2018). The Patient Safety Movement Mission. 5 de noviembre de 2018, de Patient Safety Movement.

[6] Secretaría de Salud, SSA. (2018). Conoce las Acciones Esenciales para la Seguridad del Paciente. 5 de noviembre de 2018, de Gob.mx.

[7] The Joint Comission. (2018). History of The Joint Commission. 5 de noviembre de 2018, de The Joint Comission. 